\title{
Impact of Local Site Conditions on Simulation of Non-stationary Spatial Variable Seismic Motions
}

\author{
Nassima Benmansour ${ }^{1}$, Rachid Derbal ${ }^{1,3^{*}}$, Mustapha Djafour ${ }^{1}$, Salvador Ivorra ${ }^{2}$, \\ Mohammed Matallah ${ }^{1}$ \\ ${ }^{1}$ RISk Assessment \& Management Laboratory (RISAM), University of Tlemcen, P.O. Box 230, Tlemcen, Algeria \\ ${ }^{2}$ Department of Civil Engineering, University of Alicante, San Vicente del Raspeig, Apartado 99, 03080, Spain \\ ${ }^{3}$ Department of Civil Engineering, University of Ain Temouchent, P.O. Box 284, Ain Temouchent, Algeria \\ * Corresponding author, e-mail: rachid.derbal@cuniv-aintemouchent.dz
}

Received: 12 April 2020, Accepted: 26 February 2021, Published online: 05 March 2021

\begin{abstract}
It is commonly accepted that multi-scale structures are subject to spatially variable seismic motions. This spatial variability of seismic motions is described by different intensities at different locations due to the coherency loss effect, wave passage effect and local site conditions. For multi-scale structures, the estimation of seismic excitations must consider these factors. Often, the influence of the spatial variability of seismic motion on the dynamic response of structures is performed by neglecting the site effect. In several cases, it has been observed that the high intensities of seismic motion are caused by the site amplification besides coherency loss and wave passage effects. This study aims to analyze the impact of local site conditions on seismic motions. For this purpose, a method of simulation of spatially variable seismic motions is performed. The seismic signals on the bedrock are defined by considering a target power spectral density and a coherency loss model. According to the seismic wave propagation theory, the projection of these seismic motions on the surface is realized. The results of this study show that neglecting the local site conditions induces an undervaluation of spatially variable seismic excitations.
\end{abstract}

Keywords

ground motion, spatial variability, power spectral density, coherency loss, local site conditions, simulation method

\section{Introduction}

Seismic ground motions at different sites on the surface are inevitably different due to seismic wave propagation from the source to the site. The spatially variable seismic motions are induced by three separate effects [1]. The first factor is the wave passage effect, which is due to the time delay of the arrival time of seismic waves to the different positions. The coherency loss effect is the second factor, which results from random differences in the amplitudes and phases of seismic waves. The last factor is the site effect, which due to the spatial variation of the local soil profile characteristics that affect the amplitude and frequency content of the seismic wave. For multi-scale structures like bridges, the spatial variability of seismic ground motion can significantly affect the seismic response generated internal forces. Therefore, the assumption of uniform ground motion at all supports of a multi-scale structure cannot be considered valid [2-6].

Recently, several studies on the spatially variable seismic motions, considering site effect, have been performed.
The results of these works indicate that neglecting local site effect can lead to underestimate seismic excitation for a multi-scale structure [2, 7-11]. Most of these studies have proposed methods of generating seismic signals taking into account spatial varying ground motion [9, 12-17]. The analysis of the generated signals can be performed by comparison with the target motion in terms of pick ground acceleration (PGA), pick ground displacement (PGD) and power spectrum density (PSD). Other researchers have applied these generated signals to extended structures to evaluate the influence of spatial variability of seismic ground motion on structural dynamic response. In what follows, some works on the spatial variability of seismic motion will be cited.

A method for generating spatial variable ground motions was developed by Bi et al. [8]. The seismic motions on the bedrock were assumed identical in intensity. These seismic motions are defined by a power spectral density with a target pick ground acceleration and an empirical coherency 
loss model. The site amplifications were calculated according of the one-dimensional (1-D) wave propagation theory developed by Wolf [18]. The ground motions on the surface are derived by considering the site amplification effect. The effects of spatially varying ground motion on structural responses of a frame bridge were investigated. The amplification effect is also verified depending site conditions. All results of this work show that the seismic excitation applied to a structure is conditioned by the local site conditions. So, the structural seismic response is affected by the site amplification effect.

In the study presented by Konakli and Der Kiureghian [13], a simulation method of spatially variable seismic motion is carried out by considering the coherency loss and wave passage effects. The site effect is also taking into account by some considerations. Two approaches for simulating ground motions are described. The unconditioned simulation approach considers that the motions are conditioned by a power spectral density of a subdivided seismic record. This approach gives a synthetic ground motions with uniform variability at different positions. They affirm that when analyzing responses of a multi-scale structure, the seismic variability of ground motions is uniform is crucial. While in the conditioned approach, the generation of seismic motions is established according to an earthquake record. In this case, the generated ground motions gain in variability when distance increases. The segmentation of signals is used to take into account the non-stationarity of seismic motions. Numerical example of an existing bridge is used to validate the proposed method. The soil response is modeled by two ways. In the first, the supports are concretized by one degree of freedom oscillator. While in the second case, the site response is calculated according to wave propagation theory and assuming that site is composed by one soil layer on the bedrock. The statistical characteristics of synthetic motions are compared with target theoretical models to validate the proposed method.

Based on the spectral representation method described by Deodatis [19]. Benmansour et al. [3] have proposed a technique for simulating spatially variable seismic motions. This method is based on a target earthquake record or an artificial seismic signal. The work consists to evaluate the simplified formulation given by the Algerian seismic code for bridges (RPOA) [20]. This formulation is used to analyzing bridges under spatial variability of ground motions. The results of this evaluation are compared with the results of detailed methods and with the recommendations of Eurocode 8 [21] in case of extended structures. Using this simulation technique, the generating signals can be obtained in acceleration, in velocity or in displacement directly. Several normalized bridges are used to validate the developed technique. As conclusion, they estimated that the simplified formulation proposed by RPOA overestimates the seismic demand. A modification of some provisions is necessary to gives comparable results according to deterministic method and provisions of Eurocode 8.

Zhang et al. [22] have proposed a method to generate spatial variable ground motions. In this proposed method, the non-stationarity of earthquakes, the local site conditions and the compatibility of a response spectra are considered. The spatially variable and response spectra compatible seismic motions are generated for various positions on the surface with different site configurations. The bedrock motions are supposed characterizing by $\mathrm{SH}$ wave or combination of $\mathrm{P}$ and SV waves. According a specified incident angle, these waves propagate from the bedrock until the surface. Then the seismic motions are derived by site amplification depending local site conditions. The validation of this developed method is carried out through numerical applications. Results demonstrated the compatibility of the generated non stationary seismic motions localized on the bedrock or on the surface with the bedrock response spectra and with a target loss coherency model.

A case of study of the seismic behavior of Bosphorus suspension bridge under spatially variable seismic motions is presented by Adanur et al. [7]. The impact of spatially varying earthquake on the dynamic response of this extended structure are studied. In this work, random vibration methods are performed according to spectral and spectrum response analysis. All factors of spatial varying ground motion are considered i.e. coherency loss, wave passage and local site effects. The recordings of August 17, 1999, Kocaeli, Turkey earthquake is used to describe the target power spectral density and determinate the response spectrum parameters.

The results of the random vibration analyses indicate that the choice of target power spectral density is very relevant. Indeed, the structural dynamic response is conditioned by the intensity and the frequency content of the target seismic motion.

Several other studies on the influence of local site conditions on seismic response of multi-scale structures have shown that neglecting this factor gives rise to an undervaluation of seismic demand [2, 7, 10, 11, 23]. 
This work aims to quantify the site amplification of spatially variable seismic motions. The variations of soil characteristics at a multi-scale structure supports can influence the representation of seismic excitations. For this purpose, a simulation technique of seismic motions considering all the factors of the spatial variability of seismic motion is presented. The results of site amplification of several cases are discussed. A numerical example of bridge frame is performed.

\section{Spatially variable seismic motions on the bedrock 2.1 Power spectral density model}

The spatially variable seismic motion on the bedrock at different locations is defined by a power spectral density model. The intensity in these locations is assumed the same. The filtered Tajimi-Kanai power spectral density model used on the bedrock is described by [8, 24]:

$S_{g}(\omega)=\left|H_{P}(\omega)\right|^{2} S_{0}(\omega)$,

where:

$$
\left|H_{P}(\omega)\right|^{2}=\frac{\omega^{4}}{\left(\omega_{f}^{2}-\omega^{2}\right)^{2}+\left(2 \omega_{f} \omega \xi_{f}\right)^{2}}
$$

and

$$
S_{0}(\omega)=\frac{\omega_{g}^{4}+4 \xi_{g}^{2} \omega_{g}^{2} \omega^{2}}{\left(\omega_{g}^{2}-\omega^{2}\right)^{2}+4 \xi_{g}^{2} \omega_{g}^{2} \omega^{2}},
$$

where $\omega_{f}$ and $\xi_{f}$ are the circular frequency and damping ratio of the high pass filter $\left|H_{P}(\omega)\right|^{2}$, respectively [25]. $S_{0}(\omega)$ is the Tajimi-Kanai power spectral density [26]. The parameters appearing in the Tajimi-Kanai power spectral density $S_{0}(\omega)$ are the circular frequency $\omega_{g}$ and the damping ratio $\xi_{g}$, respectively. $\Gamma$ is a scaling ratio according to the pic ground acceleration of the target seismic motion.

\subsection{Coherency loss model}

The correlation between two seismic motion regarding to the amplitudes and phase angles is described by the coherency loss function and has the following form [1]:

$$
\gamma_{j^{\prime} k^{\prime}}(\omega)=\frac{S_{j^{\prime} k^{\prime}}(\omega)}{\sqrt{S_{j^{\prime}}\left(\omega S_{k}(\omega)\right)}} .
$$

Where $S_{j^{\prime}}(\omega)$ and $S_{k^{\prime}}(\omega)$ are the power spectral density of the time histories $u_{j^{\prime}}(t)$ and $u_{k^{\prime}}(t)$, respectively. While, $S_{k}{ }^{\prime}(\omega)$ is the cross-power spectral density of $u_{j^{\prime}}(t)$ and $u_{k^{\prime}}(t)$, and $\omega$ is the circular frequency. The points $j^{\prime}$ and $k^{\prime}$ are localized on the bedrock. This coherency loss model can be written as [1]: $\gamma_{j^{\prime} k^{\prime}}(\omega)=\left|\gamma_{j^{\prime} k^{\prime}}(\omega)\right| \exp \left(-i \frac{\omega d_{j^{\prime} k^{\prime}}}{v_{a p p}}\right)$

where $d_{j^{\prime} k^{\prime}}$ is the projected horizontal distance between locations $j^{\prime}$ and $k^{\prime}$ and $v_{a p p}$ is the surface apparent velocity of waves and it is assumed constant.

\section{Spatial variable seismic motions on the surface 31 Site response amplification}

The seismic motions on the surface are derived by site amplification according to soil parameters of each location. Based on the theory of seismic wave propagation [27], the site amplification is defined by the transfer function. This function is described for shear wave propagation in a horizontal layer of soil and has the following form:

$$
\begin{aligned}
& H_{j}(i \omega)=\frac{U_{j}(i \omega)}{U_{j}^{\prime}(i \omega)}, \\
& H_{j}(i \omega)=\frac{\left(1+r_{j}-i \xi_{j}\right) \exp \left(-i \omega \tau_{j}\left(1-2 i \xi_{j}\right)\right)}{1+\left(r_{j}-i \xi_{j}\right) \exp \left(-2 i \omega \tau_{j}\left(1-2 i \xi_{j}\right)\right)},
\end{aligned}
$$

where the point $j$ ' is situated on the bedrock while the point $j$ is located on the ground surface.

$U_{j}(i \omega)$ and $U_{j^{\prime}}(i \omega)$ are the Fourier transforms of the time histories $u_{j}(t)$ and $u_{j^{\prime}}(t) . \xi_{j}$ is the site damping coefficient depending on energy dissipation; usually it has the value of $5 \% . \tau_{j}$ is the necessary time for crossing the distance between the points $j$ and $j^{\prime}$ by the seismic wave; it has the flowing relation: $\tau_{j}=h_{j} / v_{j} . r_{j}$ is the reflection coefficient for up-going waves and it is related to characteristics of the bedrock and the soil layer.

In most seismic codes, site parameters identification, mainly the velocity of shear wave propagation is almost identical. In this study, the parameters used for the sites are inspired from the site classification given by the Algeria seismic code for bridges [20] (Table 1).

Note that the predominant frequencies of the soil layer are given by:

$$
f_{k}=v_{s} k / 4 h ; k=1,3,5, \ldots,
$$

where $\boldsymbol{v}_{s}$ is the shear wave velocity, $h$ is depth of soil layer and $k$ is an impair number.

Table 1 Shear wave velocity of sites class $\boldsymbol{v}_{s}$ [20]

\begin{tabular}{lcc}
\hline Class & Description & $\boldsymbol{v}_{s}(\mathrm{~m} / \mathrm{s})$ \\
\hline S1 & Rock & $\boldsymbol{v}_{s} \geq 800$ \\
S2 & Firm & $400 \leq \boldsymbol{v}_{s} \leq 800$ \\
S3 & Medium & $200 \leq \boldsymbol{v}_{s} \leq 400$ \\
S4 & Soft & $100 \leq \boldsymbol{v}_{s} \leq 200$ \\
\hline
\end{tabular}




\section{Simulation of spatial variable seismic motions}

The simulation technique is developed based on the spectral representation method described by Shinozuka and Deodatis [28]. It is known that the simulation of seismic signals can be established by considering the unconditioned approach or the conditioned approach [13]. The difference between these two approaches lies in the definition of the target signal on the bedrock. The conditioned approach is founded on an earthquake record or an artificial signal, usually expressed in acceleration. While for the unconditioned approach, the target signal is defined by a power spectral density function corresponding to a predefined PGA and a fixed duration. In this study, the unconditioned approach is adopted to simulate spatially varying ground motions.

The following scheme resume the steps of the proposed simulation technique. A programing code is used to accomplish all these steps in loops. Each point consists to describe a step of this scheme:

1. Introduce the number of sites positions where the signals will be simulated (site number from 1 to $n$ ).

2. Formulation of the filtered Tajimi-Kanai power spectral density model $S_{g}(\omega)$ as described in Eqs. (1) to (3).

3. Choice of parameters of filtered Tajim-Kanai power spectral density function according to a given PGA and duration of the target signal (05 choices): $\omega_{f}, \xi_{f}$, $\omega_{g}, \xi_{g}$, and $\Gamma$.

4. Formulation of coherence loss model $\gamma_{j^{\prime} k^{\prime}}(\omega)$ as defined in Eq. (5).

5. Choice of the coherence loss model (11 predefined models): in this case Sobczyk model [29] is used.

6. Form the cross power spectral density matrix of seismic motions at bedrock:

$$
\begin{aligned}
& S_{j^{\prime} k^{\prime}}(\omega)=\left[\sqrt{S_{j^{\prime}}(\omega) S_{k^{\prime}}(\omega)} \gamma_{j^{\prime} k^{\prime}}(\omega)\right] \\
& j^{\prime} k^{\prime}=1,2, \ldots, n .
\end{aligned}
$$

7. Formulation of the transfer function that describes the site amplification $H_{j}(i \omega)$ as given by Eq. (7).

8. Choice of site parameters at each location (04 ground type or free choice) and at bedrock.

9. Form the cross power spectral density matrix of seismic motions on the surface $S_{j k}(\omega)$ :

$$
\begin{aligned}
& S_{j j}(\omega)=\left|H_{j}(i \omega)\right|^{2} S_{g}(\omega) ; j=1,2, \ldots, n . \\
& S_{j k}(\omega)=H_{j}(i \omega) H_{k}^{*}(i \omega) S_{j^{\prime} k^{\prime}}(i \omega) ; \\
& j \neq k \text { et } j^{\prime} \neq k^{\prime} .
\end{aligned}
$$

10. Decomposition of the cross power spectral density matrix of seismic motions on the surface $S_{j k}(\omega)$ using the Cholesky decomposition method such as:

$S_{j k}(\omega)=L(\omega) L^{T^{*}}(\omega)$.

11. Simulation of the stationary stochastic processes $g_{j}(t)$ from the real and polar elements of the matrix $L(\omega)$ such as [12]:

$g_{j}(t)=2 \sum_{m=1}^{n} \sum_{l=1}^{N}\left|L_{j m}(\omega)\right| \sqrt{\Delta \omega} \cos \left(\omega_{l}-\theta_{j m}\left(\omega_{l}\right)+\phi_{m l}\right) ;$ $j=1,2, \ldots, n$.

$\Delta \omega=\omega_{u} / N$

$\phi_{m l}$ are independent random phases uniformly distributed over the range $[0,2 \pi]$.

12. Introduce the non-stationarity to the simulated signals by multiplying them by the Jennings envelope function [26]:

$f_{j}(t)=\zeta(t) \cdot g_{j}(t) ; j=1,2, \ldots, n$,

where :

$$
\zeta(t)=\left\{\begin{array}{cc}
(t / 2)^{2} & 0 \leq t \leq 2 \\
1 & 2<t \leq 10 \\
\exp [-0.155(t-10)] & 10<t \leq T
\end{array}\right.
$$

\section{Numerical example data}

Assumed a frame bridge supported by tow piers. The span between piers has a length of $100 \mathrm{~m}$. Considering two points located on the ground surface $j$ and $k$. Their vertical projections to the bedrock are $j^{\prime}$ and $k^{\prime}$, respectively (Fig. 1).

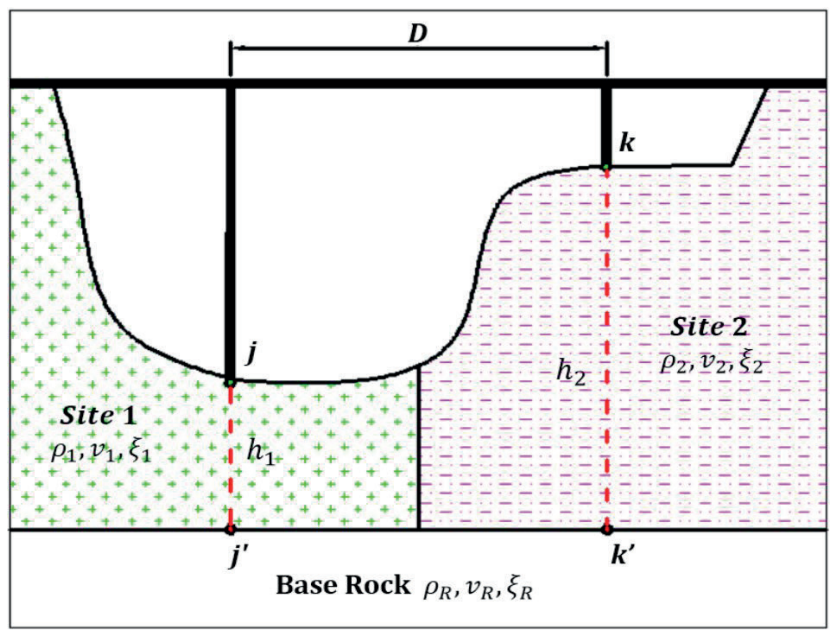

Fig. 1 Schematic view of bridge frame supported by two piers 


\subsection{Target power spectral density}

In this study, the filtered Tajimi-Kanai power spectral density used have the flowing parameters: $\omega_{f}=0.5 \pi, \xi_{f}=0.6$, $\omega_{g}=10 \pi, \xi_{g}=0.6, \Gamma=0.034 \mathrm{~m}^{2} / \mathrm{s}^{3}$. Using these values, the target seismic ground motion is defined having a pick ground acceleration (PGA) of $0.2 \mathrm{~g}$, a pick ground displacement (PGD) of $0.082 \mathrm{~m}$ and a duration equal to $20 \mathrm{~s}$ [9]. The power spectral density of seismic motions on the bedrock is given by Fig. 2 .

The considered site is composed by one layer above the bedrock with different characteristics at each bridge support. Based on previsions of the Algerian seismic code for bridges, the mean velocity of propagation of seismic shear waves has been adopted in the definition of sites parameters of this example. The parameters of bedrock and chosen sites are given by Table 2 .

\subsection{Coherency loss model}

The Sobczyk model [29] is used to define the coherency loss between the ground motions at locations at the bedrock:

$\gamma_{j^{\prime} k^{\prime}}(i \omega)=\left|\gamma_{j^{\prime} k^{\prime}}(i \omega)\right| e^{\frac{-i \omega d_{j^{\prime} k^{\prime}}}{v_{a p p}}}$,

$\gamma_{j^{\prime} k^{\prime}}(i \omega)=e^{-\beta d_{j^{\prime} k^{\prime}}} e^{-\alpha(\omega) \sqrt{d_{j k^{\prime}}}\left(\frac{\omega}{2 \pi}\right)^{2}} e^{\frac{-i \omega d_{j^{\prime k^{\prime}}}}{v_{a p p}}}$,

Where $\alpha$ is the considered incident angle of the seismic wave to the site, $\beta$ is a coefficient reflecting the level of coherency loss and $v_{a p p}$ is the apparent velocity of seismic wave. The adopted parameters are $\alpha=\pi / 3, \beta=5 \cdot 10^{-4}$ and $v_{\text {app }}=1768 \mathrm{~m} / \mathrm{s}[8]$.

\subsection{Site amplification}

The site is composed by one layer above the bedrock with different characteristics at each bridge support. Based on previsions of the Algerian seismic code for bridges, the

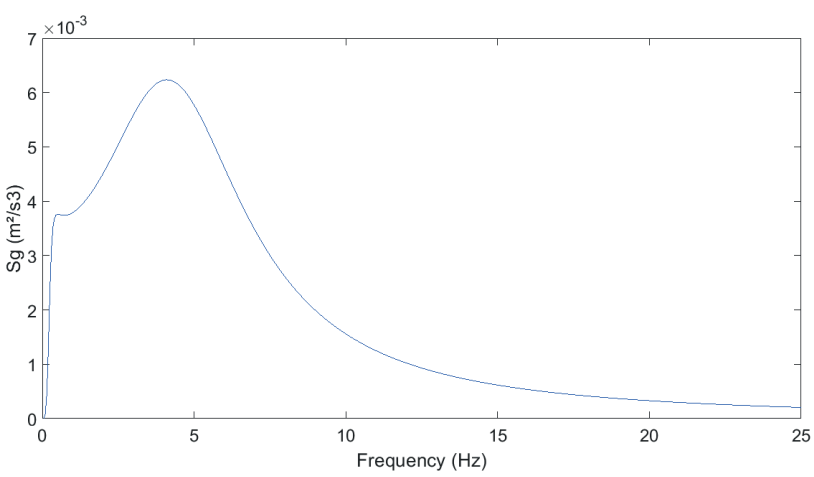

Fig. 2 Power spectral density amplitude on the bedrock in acceleration
Table 2 Parameters of sites and bedrock

\begin{tabular}{lccc}
\hline Site & Description & Parameter & Value \\
\hline \multirow{2}{*}{ Bedrock } & $\boldsymbol{\rho}_{R}$ & $3000 \mathrm{KN} / \mathrm{m}^{3}$ \\
& & $\boldsymbol{v}_{s}^{R}$ & $1500 \mathrm{~m} / \mathrm{s}$ \\
& & $\boldsymbol{\xi}_{R}$ & $5 \%$ \\
Site S1 & Firm & $\boldsymbol{\rho}_{F}$ & $2000 \mathrm{KN} / \mathrm{m}^{3}$ \\
& & $\boldsymbol{v}_{s}^{F}$ & $450 \mathrm{~m} / \mathrm{s}$ \\
Site S2 & & $\boldsymbol{\xi}_{F}$ & $5 \%$ \\
& Medium & $\boldsymbol{\rho}_{M}$ & $1800 \mathrm{KN} / \mathrm{m}^{3}$ \\
& & $\boldsymbol{v}_{s}^{M}$ & $300 \mathrm{~m} / \mathrm{s}$ \\
Site S3 & & $\boldsymbol{\xi}_{M}$ & $5 \%$ \\
& & $\boldsymbol{\rho}_{S}$ & $1500 \mathrm{KN} / \mathrm{m}^{3}$ \\
& & $\boldsymbol{v}_{s}^{S}$ & $150 \mathrm{~m} / \mathrm{s}$ \\
& & $\boldsymbol{\xi}_{S}$ & $5 \%$ \\
\hline
\end{tabular}

mean velocity of propagation of seismic shear waves has been adopted in the definition of sites parameters of this example. $\rho$ is the density, $v_{s}$ is the shear wave velocity and $\xi$ is the damping ratio. In the Table 2 , the superscripts $R, F$, $M$ and $S$ represent the bedrock, firm site, medium site and soft site, respectively.

\section{Results and discussion}

\subsection{Effect of soil layer depth and site type}

The effect of soil depth on the amplification of generated seismic motions are investigated. Five different soil depths and three site class are adopted. The sites class are S1 (Firm), S2 (Medium) and S3 (Soft). The depths of soil layer are $0,10,20,30$ and $50 \mathrm{~m}$. Note that the span length is $100 \mathrm{~m}$.

For each site class, the evolution of amplification is plotted for different depth. As shown in Figs. 3 to 5, different soil depths lead to different transfer functions. The peaks occur at the corresponding vibration modes of the sites.

Adopting a firm site under the two piers of bridge frame, the predominant $F_{r}$ and resonant frequencies and $F_{k=1}$ for different depth of site layer are given in Table 3. The amplitude of amplification spectra for a firm site is about 2.83 .

While considering a medium site at $j$ and $k$, the predominant and resonant frequencies for different depth of site layer are given in Table 4. The amplitude of amplification spectra for a medium site is about 3.66.

Assuming soft sites at $j$ and $k$, the predominant and resonant frequencies for different depth of site layer are given in Table 5. The amplitude of amplification spectra for a soft site is about 4.91 .

From Figs. 3, 4 and 5, we can conclude that deeper is the soil layer, more flexible is the site, and lower is the predominant frequency. 


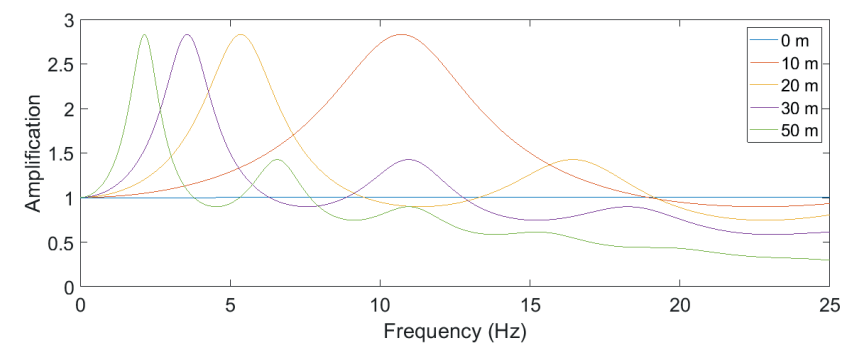

Fig. 3 Site amplification spectra considering firm sites

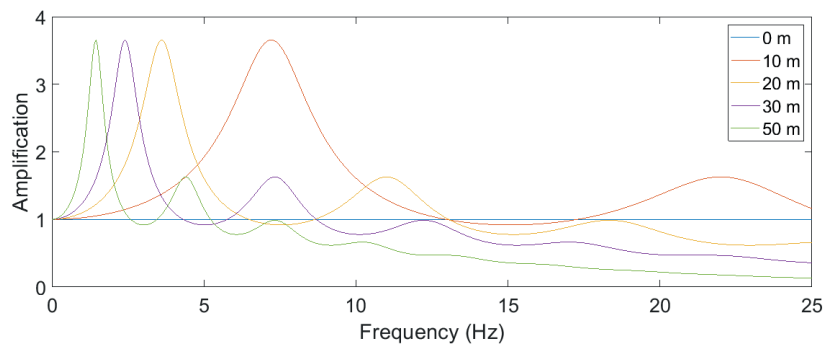

Fig. 4 Site amplification spectra considering medium sites

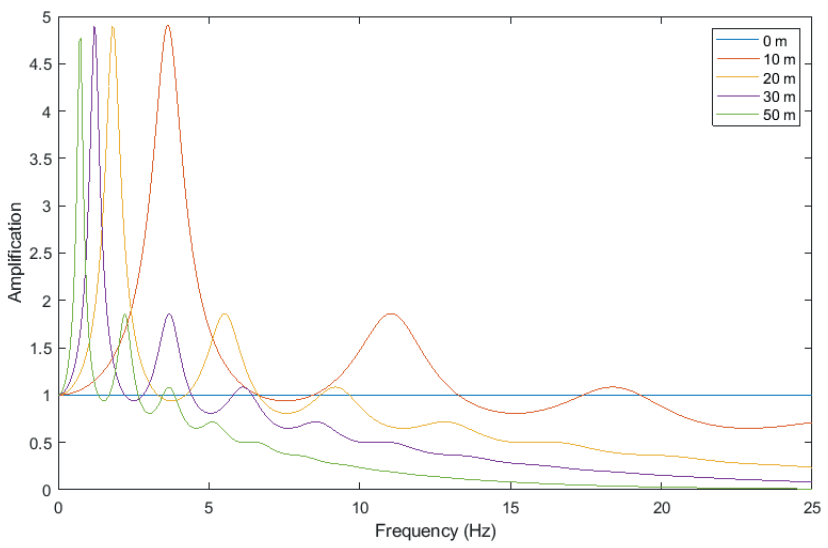

Fig. 5 Site amplification spectra considering soft sites

Table 3 Predominant frequency considering firm sites

\begin{tabular}{lccccc}
\hline Depth $(\mathrm{m})$ & 0 & 10 & 20 & 30 & 50 \\
$F_{r}(\mathrm{~Hz})$ & 0 & 10.70 & 5.35 & 3.55 & 2.20 \\
$F_{k}=1(\mathrm{~Hz})$ & 0 & 11.25 & 5.62 & 3.75 & 2.25 \\
\hline
\end{tabular}

Table 4 Predominant frequency considering medium sites

\begin{tabular}{lccccc}
\hline Depth $(\mathrm{m})$ & 0 & 10 & 20 & 30 & 50 \\
$F_{r}(\mathrm{~Hz})$ & 0 & 7.20 & 3.60 & 2.40 & 1.45 \\
$F_{k}=1(\mathrm{~Hz})$ & 0 & 7.50 & 3.75 & 2.50 & 1.50 \\
\hline
\end{tabular}

Table 5 Predominant frequency considering soft sites

\begin{tabular}{llcccc}
\hline Depth $(\mathrm{m})$ & 0 & 10 & 20 & 30 & 50 \\
$F_{r}(\mathrm{~Hz})$ & 0 & 3.65 & 1.80 & 1.20 & 0.75 \\
$F_{k}=1(\mathrm{~Hz})$ & 0 & 3.75 & 1.87 & 1.25 & 0.75 \\
\hline
\end{tabular}

This conclusion is supported by the Figs. 6 and 7. Where Fig. 6 represent the amplitude of site amplification for a soft site at point $\mathrm{j}$ and a medium site at point $\mathrm{k}$ with a depth

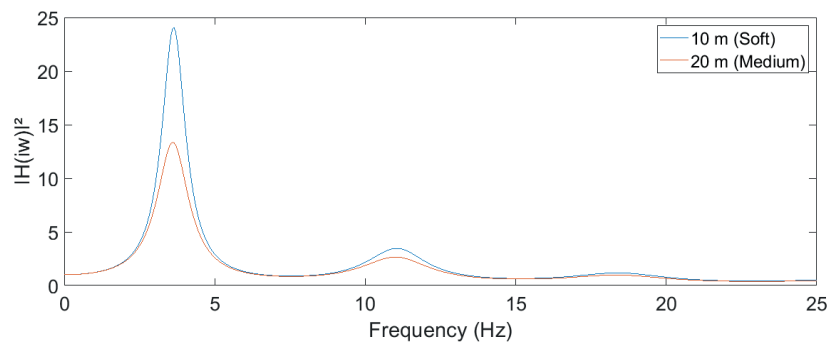

Fig. 6 Site amplification spectra (site $j$ : soft, $h=10 \mathrm{~m}$; site $k$ : medium, $h=20 \mathrm{~m})$

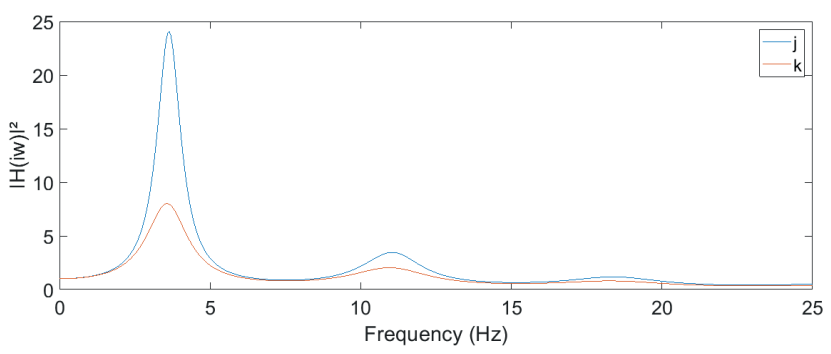

Fig. 7 Site amplification spectra (site $j$ : soft, $h=10 \mathrm{~m}$; site $k$ : medium,

$$
h=30 \mathrm{~m})
$$

of $10 \mathrm{~m}$ and $20 \mathrm{~m}$ respectively. Fig. 7 represent the amplitude of site amplification for a soft site at point $j$ and a firm site at point $k$ with a depth of $10 \mathrm{~m}$ and $30 \mathrm{~m}$ respectively. In this both particular cases, the predominant frequencies are almost identical for the sites at $j$ and $k$ (Tables 6 and 7).

\subsection{Study case example}

In the following, we adopt a different site conditions under the two piers of bridge frame the parameters of bedrock and sites adopted for the simulation of spatially variable ground motions are given by Table 8 . For this case, the amplitude of site amplification is illustrated in Fig. 8.

First, the simulation of seismic signals is performed without considering spatial variable ground motion i.e., neglecting the effects of coherency loss, wave passage and

Table 6 Predominant frequency (site $j$ : soft, $h=10 \mathrm{~m}$; site $k$ : medium,

\begin{tabular}{lcc}
\multicolumn{3}{c}{$h=20 \mathrm{~m})$} \\
\hline Sites & Soft & Medium \\
\hline Depth $(\mathrm{m})$ & 10 & 20 \\
$F_{r}(\mathrm{~Hz})$ & 3.65 & 3.60 \\
$F_{k}=1(\mathrm{~Hz})$ & 3.75 & 3.75 \\
\hline
\end{tabular}

Table 7 Predominant frequency (site $j$ : soft, $h=10 \mathrm{~m}$; site $k$ : medium,

\begin{tabular}{lcc}
\multicolumn{3}{c}{$h=30 \mathrm{~m})$} \\
\hline Sites & Soft & Firm \\
\hline Depth $(\mathrm{m})$ & 10 & 30 \\
$F_{r}(\mathrm{~Hz})$ & 3.65 & 3.55 \\
$F_{k}=1(\mathrm{~Hz})$ & 3.75 & 3.75 \\
\hline
\end{tabular}


Table 8 Parameters of bedrock and sites adopted

\begin{tabular}{lccc}
\hline & Description & Parameter & Value \\
\hline \multirow{2}{*}{ Site 1 } & $\rho_{1}$ & $1800 \mathrm{KN} / \mathrm{m}^{3}$ \\
& Medium & $v_{1}$ & $300 \mathrm{~m} / \mathrm{s}$ \\
& $\xi_{1}$ & $5 \%$ \\
& & $h_{1}$ & $10 \mathrm{~m}$ \\
Site 2 & $\rho_{2}$ & $2000 \mathrm{KN} / \mathrm{m}^{3}$ \\
& & $v_{2}$ & $450 \mathrm{~m} / \mathrm{s}$ \\
& & $\xi_{2}$ & $5 \%$ \\
Bedrock & $h_{2}$ & $30 \mathrm{~m}$ \\
& & $\rho_{\text {sub }}$ & $1500 \mathrm{KN} / \mathrm{m}^{3}$ \\
& & $v_{\text {sub }}$ & $150 \mathrm{~m} / \mathrm{s}$ \\
& & $\xi_{\text {sub }}$ & $5 \%$ \\
\hline
\end{tabular}

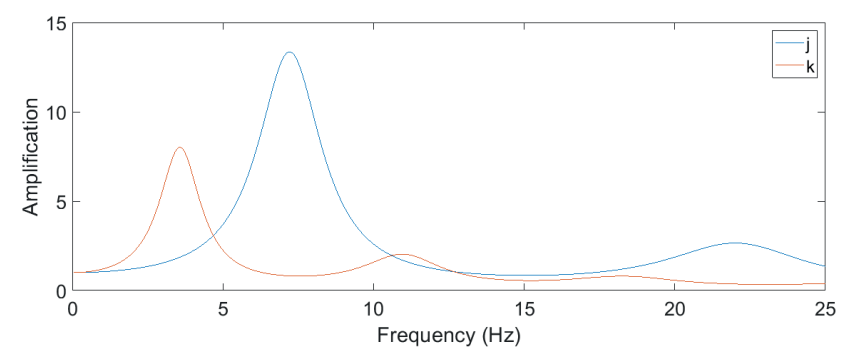

Fig. 8 Site amplification spectra for case studied

site effect. The simulated seismic motions are supposed completely coherent i.e. uniform. This step serves to validate the simulation technique of seismic signals (Figs. 9-10).

Then, the signals are simulated at the bedrock i.e. taking into account the loss coherency and wave passage effects. The site effect is neglected in this case. The accelerations and displacements generated are given in Figs. 11 and 12, respectively. The simulated accelerations and displacements at the site $k^{\prime}$ have a PGA $=0.27 \mathrm{~g}$ and a PGD $=0.100 \mathrm{~m}$, respectively. While the simulated signal at the site $j^{\prime}$ keep the same PGA and PGD as the uniform ground motions.

The power spectral density of simulated bedrock accelerations is compared with the target power spectral density (filtered Tajimi-Kanai PSD) and it is illustrated in Fig. 13. It is observed that both PSD of the simulated signals are comparable.

Finally, the simulated ground motions on the bedrock are projected at the ground surface by amplification according the parameters of site and the depth of soil layer under each pier. The simulated accelerations and displacements at the ground surface are given by Figs. 14 and 15, respectively.

The simulated signal at the site $j$ has a $\mathrm{PGA}=0.46 \mathrm{~g}$ and a $\mathrm{PGD}=0.119 \mathrm{~m}$. While at the site $k$, the $\mathrm{PGA}=0.45 \mathrm{~g}$ and the $\mathrm{PGD}=0.116 \mathrm{~m}$. The effect of site amplification on the seismic motions on the surface is clearly visible in this case.
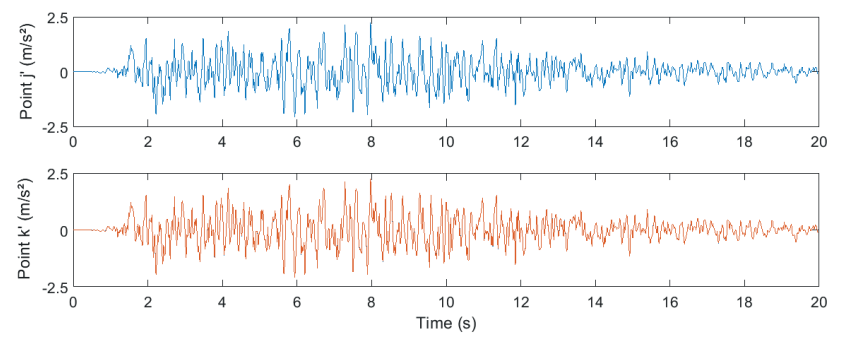

Fig. 9 Simulated uniform seismic motions in acceleration
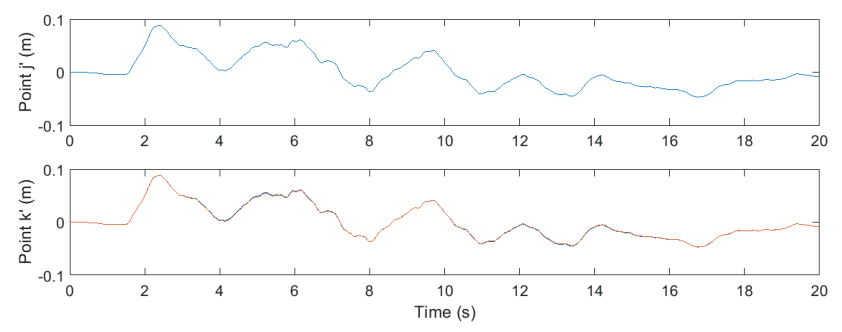

Fig. 10 Simulated uniform seismic motions in displacement
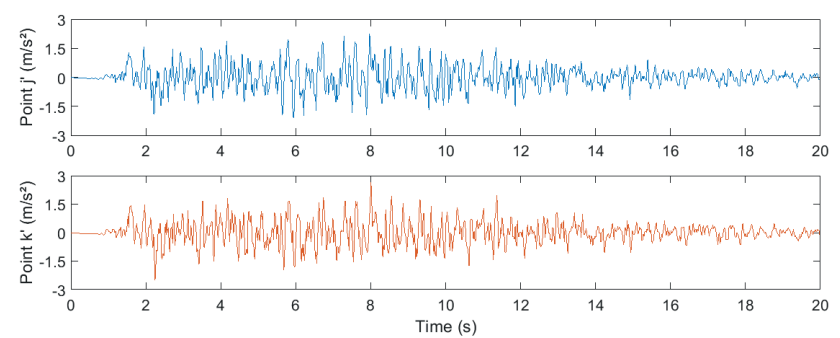

Fig. 11 Simulated seismic motions in acceleration on the bedrock

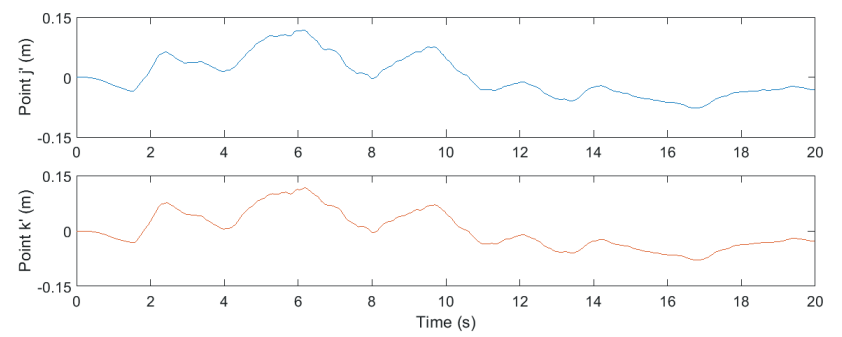

Fig. 12 Simulated seismic motions in displacement on the bedrock

As showed in Fig. 16, the power spectral density of simulated ground surface accelerations is compared with the target Tajimi-Kanai power spectral density. The both PSD of simulated signals are superior to the target PSD with different predominant frequencies.

\section{Conclusions}

A seismic motions simulation technique considering all the factors of the spatial variability of seismic motion is developed. A numerical example of bridge frame is performed. The site amplification of several cases is discussed. Results of the effects of soil layer depth and site class on the site amplification are investigated. These results demonstrate 

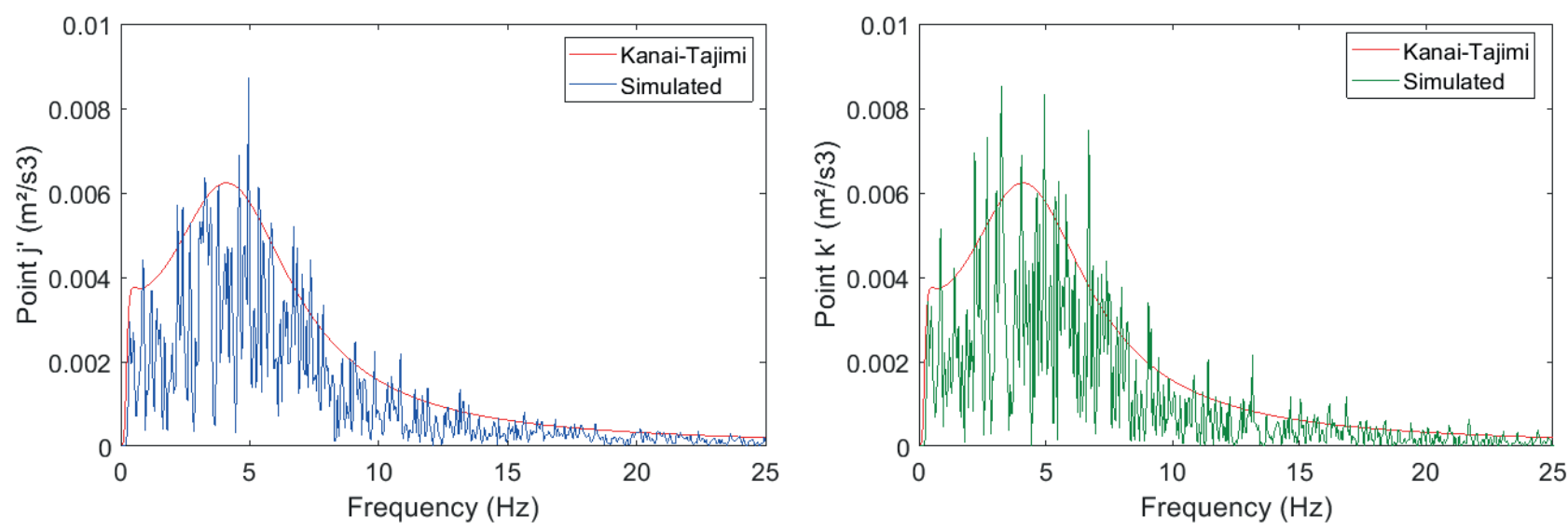

Fig. 13 Comparison between the power spectral density of simulated bedrock accelerations and the target power spectral density
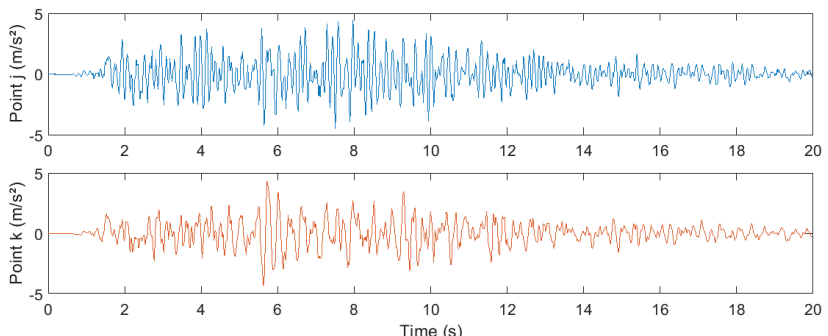

Fig. 14 Simulated seismic motions in acceleration at the surface

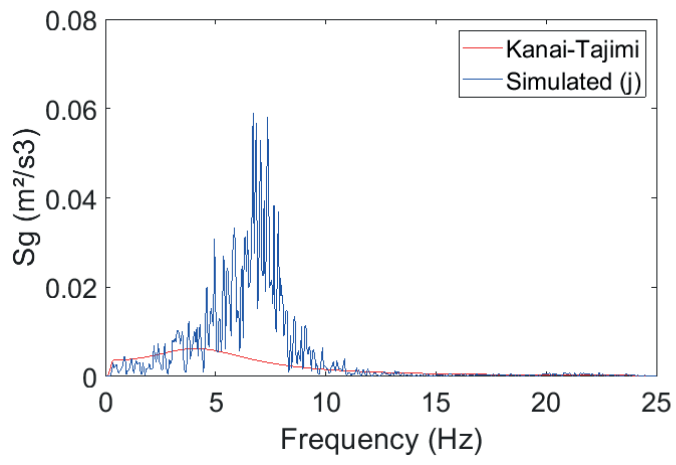

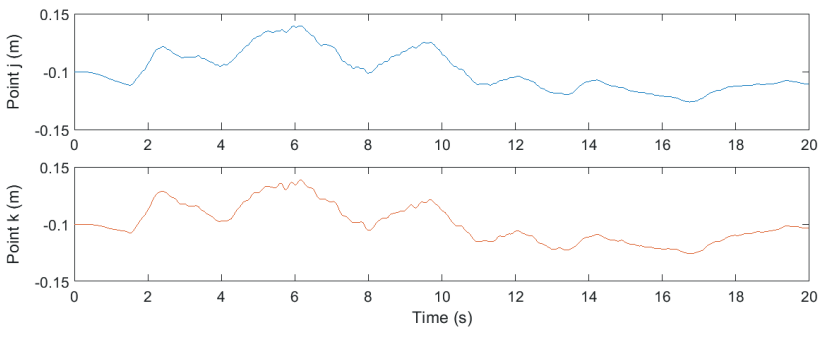

Fig. 15 Simulated seismic motions in displacement at the surface

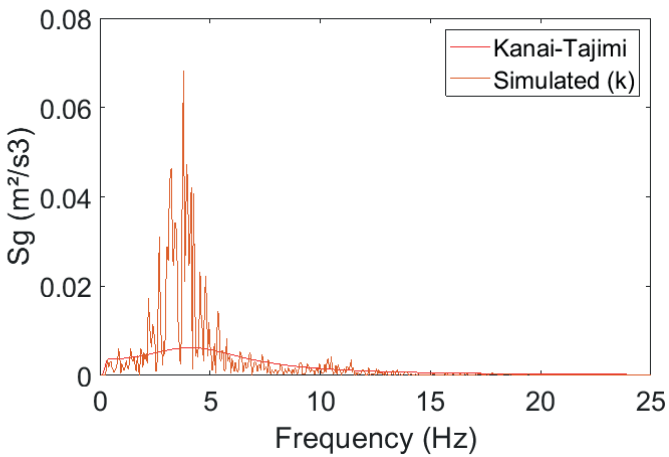

Fig. 16 Comparison between the power spectral density of simulated surface ground accelerations

that deeper is the soil layer, more flexible is the site, and lower is the predominant frequency. Two particular cases are presented where the predominant frequencies are almost identical at both sites.

Adopting uniform case, the simulated ground motions at different sites have a perfect match. This case validates the simulation technique of seismic signals. The simulated ground motions are slightly amplified because of the coherency loss resulting from the propagation of seismic waves. The effect of site amplification on the seismic motions on the surface is predominant. In fact, neglecting local site conditions lead to an undervaluation of seismic input applied to multi-scale structures.

\section{Acknowledgement}

Authors would acknowledge the support of the Department of Civil Engineering, University of Alicante, Spain to develop this work as part of research interchange between the University of Alicante and the Ministry of High Education and Scientific Research of Algeria. 


\section{References}

[1] Der Kiureghian, A. "A Coherency Model for Spatially Varying Ground Motions", Earthquake Engineering and Structural Dynamics, 25, pp. 99-111, 1996.

https://doi.org/10.1002/(SICI)1096-9845(199601)25:1<99::AIDEQE540>3.0.CO;2-C

[2] Derbal, R., Benmansour, N., Djafour, M., Matallah, M., Ivorra, S. "Viaduct seismic response under spatial variable ground motion considering site conditions", Earthquakes and Structures, 17(6), pp. 557-566, 2019.

https://doi.org/10.12989/eas.2019.17.6.557

[3] Benmansour, N., Djafour, M., Bekkouche, A., Zendagui, D., Benyacoub, A. "Seismic response evaluation of bridges under differential ground motion: a comparison with the new Algerian provisions", European Journal of Environmental and Civil Engineering, 16(7), pp. 863-881, 2012.

https://doi.org/10.1080/19648189.2012.681951

[4] Benmansour, N., Djafour, M., Zendagui, D., Bekkouche, A. "Non linear dynamic analysis of bridge to spatially variable multiple support excitations", presented at 9th International Conference on Urban Earthquake Engineering/ 4th Asia Conference on Earthquake Engineering, Tokyo Institute of Technology, Tokyo, Japan, March, 6-8, 2012.

[5] Djafour, M., Meddane, N., Derbal, R., Megnounif, A., Zendagui, D., Bekkouche, A. "Response of a gravity arch dam to spatially varying earthquake ground motion", presented at 8th National Conference on Earthquake Engineering, San Francisco, CA, USA, Apr. 18-22, 2006.

[6] Djafour, M., Meddane, N., Derbal, R., Zendagui, D., Bekkouche, A. "Étude du comportement dynamique d'un barrage-voûte face au mouvement sismique différentiel" (Study of the dynamic behavior of an arch dam in the face of differential seismic movement), presented at 18th French Congress of Mechanics, Grenoble, France, Aug. 27-31, 2007. (in French)

[7] Adanur, S., Altunişik, A. C., Soyluk, K., Bayraktar, A., Dumanoğlu, A. A. "Multiple-support seismic response of Bosporus Suspension Bridge for various random vibration methods", Case Studies in Structural Engineering, 5, pp. 54-67, 2016.

https://doi.org/10.1016/j.csse.2016.04.001

[8] Bi, K., Hao, H., Ren, W. "Response of a frame structure on a canyon site to spatially varying ground motions", Structural Engineering and Mechanics, 36(1), pp. 111-127, 2010.

https://doi.org/10.12989/sem.2010.36.1.111

[9] Bi, K., Hao, H. "Modelling and simulation of spatially varying earthquake ground motions at sites with varying conditions", Probabilistic Engineering Mechanics, 29, pp. 92-104, 2012. https://doi.org/10.1016/j.probengmech.2011.09.002

[10] Derbal, R., Benmansour, N., Djafour, M. "Influence de l'effet de site sur le comportement dynamique des ponts" (Influence of the site effect on the dynamic behavior of bridges), presented at 23rd French Congress of Mechanics, Lille, France, Aug. 28-Sept. 1, 2017. (in French)

[11] Derbal, R., Benmansour, N., Djafour, M. "Impact of Spatial Variability of Earthquake Ground Motion on Seismic Response of a Railway Bridge", International Journal of Computational Methods and Experimental Measurements, 6(5), pp. 910-920, 2018.

https://doi.org/10.2495/CMEM-V6-N5-910-920
[12] Benmansour, N. "Effet de la Variabilité Spatiale du Mouvement Sismique sur le Comportement Dynamique des Ponts" (Effect of Spatial Variability of Seismic Motion on the Dynamic Behavior of Bridges), Doctoral Thesis, University Abou Bekr Belkaid, 2013. (in French)

[13] Konakli, K., Der Kiureghian, A. "Simulation of spatially varying ground motions including incoherence, wave $\square$ passage and differential site $\square$ response effects", Earthquake Engineering and Structural Dynamics, 41, pp. 495-513, 2012.

https://doi.org/10.1002/eqe.1141

[14] Wu, Y., Gao, Y., Zhang, N., Zhang, F. "Simulation of Spatially Varying Non-Gaussian and Nonstationary Seismic Ground Motions by the Spectral Representation Method", Journal of Engineering Mechanics, 144(1), Article No. 04017143, 2018. https://doi.org/10.1061/(ASCE)EM.1943-7889.0001371

[15] Rodda, G. K., Basu, D. "Spatial variation and conditional simulation of seismic ground motion", Bulletin of Earthquake Engineering, 16, pp. 4399-4426, 2018. https://doi.org/10.1007/s10518-018-0397-6

[16] Rodda, G. K., Basu, D. "Spatially correlated vertical ground motion for seismic design", Engineering Structures, 206, Article No. 110191, 2020.

https://doi.org/10.1016/j.engstruct.2020.110191

[17] Falamarz-Sheikhabadi, M. R., Zerva, A. "Two uncertainties in simulating spatially varying seismic ground motions: incoherency coefficient and apparent propagation velocity", Bulletin of Earthquake Engineering, 16, pp. 4427-4441, 2018. https://doi.org/10.1007/s10518-018-0385-x

[18] Wolf, J. P. "Dynamic Soil-Structure Interaction", Prentice-Hall, Englewood Cliffs, NJ, USA, 1985.

[19] Deodatis, G. "Non-stationary stochastic vector processes: seismic ground motion applications", Probabilistic Engineering Mechanics, 11(3), pp. 149-167, 1996. https://doi.org/10.1016/0266-8920(96)00007-0

[20] RPOA (Algerian Seismic Code for Bridges) "Seismic Rules Applicable to the Field of Structures", Regulatory Technical Document (DTR), Ministry of Public Works and Transport, El Biar, Algeria, 2008. (in French)

[21] CEN "Eurocode 8: Design of Structures for Earthquake Resistance", European Committee for Standardization, Brussels, Belgium, 2004.

[22] Zhang, D.-Y., Liu, W., Xie, W.-C., Pandey, M. D. "Modeling of spatially correlated, site-reflected, and nonstationary ground motions compatible with response spectrum", Soil Dynamics and Earthquake Engineering, 55, pp. 21-32, 2013. https://doi.org/10.1016/j.soildyn.2013.08.002

[23] Shiravand, M. R., Parvanehro, P. "Spatial variation of seismic ground motion effects on nonlinear responses of cable stayed bridges considering different soil types", Soil Dynamics and Earthquake Engineering, 119, pp. 104-117, 2019. https://doi.org/10.1016/j.soildyn.2019.01.002

[24] Tajimi, H. "A Statistical Method of Determining the Maximum Response of a Building Structure during an Earthquake", Proceeding of 2nd World Conference on Earthquake Engineering, Tokyo, Japan, 1960, pp. 781-796. 
[25] Clough, R. W., Penzien, J. "Dynamics of Structures", McGraw Hill, New York, NJ, USA, 1993.

[26] Jennings, P. C., Housner, G. W., Tsai, N. C. "Simulated Earthquake Motions", California Institute of Technology, Pasadena, CA, USA, Rep. EERL-02, 1968. [online] Available at: https://authors.library. caltech.edu/26504/

[27] Şafak, E. "Discrete-Time Analysis of Seismic Site Amplification", Journal of Engineering Mechanics, 121(7), pp. 801-809, 1995. https://doi.org/10.1061/(ASCE)0733-9399(1995)121:7(801)
[28] Shinozuka, M., Deodatis, G. "Simulation of Stochastic Processes by Spectral Representation", Applied Mechanics Reviews, 44(4), pp. 191-204, 1991. https://doi.org/10.1115/1.3119501

[29] Sobczyk, K. "Stochastic Wave Propagation", Kluwer Academic Publishers, Dordrecht, The Netherlands, 1991. 